\title{
Tuberculosis Unseen - Missed Opportunities in Diagnosis
}

\author{
Aaron C. Miller*, Linnea A. Polgreen and Philip M. Polgreen \\ University of lowa, lowa City, IA, USA
}

\section{Objective}

To estimate the potential number of Tuberculosis (TB) cases that occur in inpatient and emergency department settings that are missed, diagnosed as something else, go untreated and return to the community, prior to receiving a correct diagnosis of TB. We analyze inpatient and emergency department records from the state of California from 2005-2011.

\section{Introduction}

Historically, patients with TB have often been diagnosed late ${ }^{1}$ or after death ${ }^{2}$. This delay in diagnosis often occurs because TB is misdiagnosed as an alternative respiratory illness (RI), such as pneumonia ${ }^{1}$. TB infected patients that are not correctly diagnosed when initial symptoms occur may spread infection to others in both healthcare settings and the community.

\section{Methods}

HCUP state inpatient and emergency department records for the state of California were extracted for patients with multiple stays between 2005 and 2011. Records containing an ICD-9 diagnosis code beginning with $010,011,012$, and 018 were used to identify patients with a TB diagnosis. Next, we searched for visits that preceded the patient's TB diagnosis. Patient records following the first TB diagnosis and those containing a prior alternative TB diagnosis (e.g. ICD-9 codes starting with 015 - TB of the bones and joints) were excluded. We defined a potential missed diagnosis (PMD) to have occurred if: (1) a RI was indicated in the previous visit and (2) that visit occurred in an event window of 5-90 days directly prior to the admission containing the initial TB diagnosis. If the previous visit occurred less than 5 days prior to the TB associated visit, it was excluded because there is a time lag between TB testing and diagnosis.

Finally, in order to determine if the RI's in a visit preceding a TB diagnosis represent a PMD rather than a correct but coincident RI diagnoses, the occurrence of such events was compared between patients with and without an initial TB diagnosis. Odds ratios were computed for the probability of a RI occurring in a previous stay falling in the same event window between patients with and without a TB diagnosis. Multivariate logistic regression was also used to estimate this odds ratio while controlling for patient characteristics.

\section{Results}

A total of 6,664 initial cases of TB were identified, 3,164 of which had a RI in the visit directly preceding their initial TB diagnosis. Of the initial TB cases identified, approximately $23.33 \%$ had a PMD, defined by a RI. The rate of PMDs increased with larger time windows: PDE rates were $19.75 \%, 25.33 \%, 28.45 \%$, to $32.5 \%$, when $60,120,270$ and 360 day time windows were used, respectively.

In comparison to the non-TB patients, those with an initial TB diagnosis were far more likely to experience a $\mathrm{RI}$ in a previous visit (OR 3.86, CI 3.58-4.16), and only $8.27 \%$ of the previous visits in the non-TB population contained a RI. After controlling for patient characteristics, the odds of an initial TB diagnosis having a previous visit containing a RI was 2.77 (CI 2.55-3.00) times the odds of a non-TB visit.

\section{Conclusions}

Our results indicated that misdiagnosis of TB may be occurring in more than $20 \%$ of TB patients diagnosed in inpatient and emergency department settings. While some of these events likely represent coincident RIs rather than PMDs, patients with an initial TB diagnosis were far more likely to experience a RI in the months leading up to diagnosis than non-TB patients. Moreover, given that RIs are often diagnosed and treated in outpatient settings, even more misdiagnoses may be occurring than can be observed in the data used here. These findings call for an increased awareness in TB surveillance, especially to prevent healthcare associated spread of TB.

\section{Keywords}

Tuberculosis; Misdiagnosis; Disease surveillance

\section{References}

1. Wang J, Hsueh P, Jan I, Liaw Y, Yang P, Luh K. Empirical treatment with a fluoroquinolone delays the treatment for tuberculosis and is associated with a poor prognosis in endemic areas. Thorax. 2006; 61(10)903-8.

2. Bobrowitz I. Active Tuberculosis Undiagnosed Until Autopsy. Am. J. Med. 1982; 72(4): 650-8.

\section{*Aaron C. Miller}

E-mail: aaron-miller@uiowa.edu 